\title{
Bronchial valve treatment for pulmonary air leak after anatomical lung resection for cancer
}

\author{
Christophe A. Dooms ${ }^{2}$, Herbert Decaluwe², Jonas Yserbyt ${ }^{1}$, Paul De Leyn², \\ Dirk Van Raemdonck ${ }^{2}$ and Vincent Ninane $e^{1,3}$
}

Affiliations: ${ }^{1}$ Respiratory Division, University Hospitals Leuven, Leuven, ${ }^{2}$ Dept of Thoracic Surgery, University Hospitals Leuven, Leuven, and ${ }^{3}$ Chest Service Saint-Pierre Hospital Brussels, Brussels, Belgium.

Correspondence: C.A. Dooms, University Hospitals Leuven, Respiratory Division, Herestraat 49, B-3000 Leuven, Belgium. E-mail: christophe.doomsduzleuven.be

ABSTRACT A persistent post-operative pulmonary expiratory air leak after an anatomical pulmonary resection is usually managed conservatively, but can be associated with significant morbidity and increased costs. The use of bronchial valves is a minimally invasive method that may be an effective and safe treatment in this setting.

In a prospective study, the clinical efficacy of intrabronchial valve treatment in patients with a prolonged persistent pulmonary air leak after anatomical surgical resection for cancer was investigated.

10 out of 277 patients with anatomical pulmonary resection for cancer were included, and $90 \%$ were scheduled for valve treatment. We demonstrated an air leak cessation at a median of 2 days after valve placement, which resulted in chest tube removal at a median of 4 days after valve placement. Elective removal of the intrabronchial valves could be safely planned 3 weeks after valve implantation. Lung function alteration associated with airway occlusion by valves was limited.

Intrabronchial valve treatment with the aid of a digital thoracic drainage system is an effective and safe therapy for patients with a prolonged pulmonary air leak after anatomical lung resection for cancer.

@ERSpublications

Bronchial valve placement is an effective and safe treatment for a pulmonary air leak after lung resection http://ow.ly/swou7 


\section{Introduction}

In the USA, $\sim 50000$ patients each year are potential candidates for a surgical resection for early-stage lung cancer [1]. A prolonged pulmonary air leak in this setting is independently associated with prolonged hospital length of stay, decreased patient satisfaction and increased morbidity or post-operative complications, and adds significantly to the healthcare cost $[2,3]$. The use of bronchial valves was first considered a potential effective salvage procedure for the treatment of a persistent pulmonary air leak in patients who were not suitable candidates for any other surgical treatment $[4,5]$. Retrospective case series provided scientific evidence that removable bronchial valves are a safe and effective intervention for alveolar-pleural fistula with persistent pulmonary air leak $[6,7]$. Limitations of reported case studies and series are their retrospective nature, chest tube monitoring mainly based on a subjective assessment of the air leak reduction, and heterogeneity of disorders causing the persistent air leak. We therefore decided to perform a prospective study objectively evaluating the efficacy and safety of airway closure using intrabronchial valves for the treatment of a persistent post-operative air leak after anatomical lung resection for cancer. For this purpose, we assessed air leak monitoring during and after intrabronchial valve treatment using a digital thoracic drainage system, and respiratory function alteration linked to temporary airway closure.

\section{Methods}

This is a prospective, observational, single centre study (registered at www.clinicaltrials.gov with identifier number NCT01451359) evaluating the efficacy of intrabronchial valve treatment in consecutive patients with a prolonged persistent pulmonary air leak after anatomical surgical resection for cancer.

\section{Patients}

Inclusion criteria were prolonged ( $10 \pm 3$ days post-operative) persistent air leak refractory to conventional treatment (such as prolonged drainage and/or peri-operative chemical pleurodesis), anatomical lung resection (such as segmentectomy, (bi)lobectomy or sleeve lobectomy), air leak after antero/posterolateral thoracotomy or video-assisted thoracoscopy (VATS), and expiratory air leak of any size $\geqslant 100 \mathrm{~mL} \cdot \mathrm{min}^{-1}$ measured by a digital thoracic drainage system (Thopaz; Medela AG, Baar, Switzerland). Patients were excluded whenever the post-operative prolonged air leak was present for $>13$ days, pneumonectomy or nonanatomical lung resection was performed, lung resection for an indication other than cancer was performed, a previous Heimlich valve was applied, in case of empyema, or whenever the patient was unable to give an informed consent. A thoracic surgeon assessed the patients during their early post-operative period. A "diagnostic balloon occlusion test" performed by flexible bronchoscopy under local anaesthesia was required in order to assess whether the air leak could be stopped and/or whether the patient could tolerate the provocative (sub)lobar occlusion. All patients provided written informed consent after study approval by a Local Institutional Review Board (University Hospitals Leuven, Leuven, Belgium; B32220096119).

\section{Study procedures}

The chest tube was connected to a digital thoracic drainage system (Thopaz) continuously measuring and displaying the air leak, while the suction level was standardised at $-8 \mathrm{cmH}_{2} \mathrm{O}$ in all patients. During the scheduled diagnostic balloon occlusion test by flexible bronchoscopy under local anaesthesia with moderate sedation, a balloon catheter was passed through the working channel of the bronchoscope, placed in the suspected segmental bronchi and inflated until complete occlusion of segmental bronchi occurred. A sequential balloon occlusion of segmental or lobar airways was performed with the aim to stop truly the air leak (defined as air leak $<20 \mathrm{~mL} \cdot \mathrm{min}^{-1}$ as displayed on the Thopaz system) and thus attribute the air leak to the particular segment tested, but also to test whether the patient could clinically tolerate a (sub)lobar occlusion similar to that following intrabronchial valve treatment. Only patients with an identified target lobe/segment for valve treatment and tolerable airway occlusion were considered for subsequent intrabronchial valve treatment.

Intrabronchial valve treatment was performed using the Spiration Inc. (Olympus Respiratory America, Redmond, WA, USA) intrabronchial valve and delivery catheter (IBV Valve System; Olympus Respiratory America), according to a predefined protocol of IBV sizing and placement. The valve treatment was scheduled on the same day or the day after the diagnostic balloon occlusion test bronchoscopy. The procedure was performed under general anaesthesia with an 8-French endotracheal tube applying inspiratory positive pressure mechanical ventilation (standardised intermittent positive pressure ventilation settings: tidal volume $8 \mathrm{~mL} \cdot \mathrm{kg}^{-1}$, frequency 12 breaths per minute, inspiratory oxygen fraction $50 \%$ and no positive end-expiratory pressure). The first step of valve placement was airway sizing using the IBV airway sizing kit, to determine the appropriate valve size $(5,6$ or $7 \mathrm{~mm})$. Once the appropriate valve was loaded into an IBV catheter, the catheter was advanced through the working channel of the bronchoscope into the 
target airway segment where valve deployment was performed. A continuous digital air leak assessment before and during valve placement enabled a logical and stepwise occlusion of (sub)segmental bronchi until air leak cessation was obtained during digital air leak monitoring. The complete description of the procedure is reported elsewhere [8]. The patient was extubated in the endoscopy suite once the last valve was inserted. In the recovery room, the air leak was digitally assessed during spontaneous breathing and a chest radiograph was performed to assess the lung inflation status. The Thopaz system is kept at $-8 \mathrm{cmH}_{2} \mathrm{O}$ suctioning and air leak flow is continuously measured enabling the treating physician to decide upon chest tube removal once the air leak is stopped for $\geqslant 8 \mathrm{~h}$.

All patients were scheduled to have a chest radiograph and pulmonary function test 3-4 weeks after endoscopic valve placement. After these measurements, a pre-planned removal of all intrabronchial valves was scheduled between day 21 and day 28 after valve placement, and was performed during a flexible bronchoscopy under local anaesthetic with moderate sedation. Within 7 days of removal of the valves, a control chest radiograph and pulmonary function test were performed.

\section{Outcome measures}

The primary study end-point is the clinical efficacy on air leak cessation allowing chest tube removal. Other evaluations included: avoidance of Heimlich valve; avoidance of additional surgical intervention and safety issues including complications related to IBV treatment (e.g. pulmonary infection, valve migration, pneumothorax requiring treatment and respiratory insufficiency); evaluation of consequences of airway closure on pulmonary function; the direct cost related to the device used; and, finally, timing of bronchial valve removal.

\section{Statistical analysis}

Data analysis was performed using the GraphPad Prism 4 statistical software package (GraphPad Software, Inc., San Diego, CA, USA). Differences within each group at different time-points were tested using a paired t-test. A p-value $<0.05$ was considered as significant.

\section{Results}

Study population and clinical and procedural characteristics

Study population and clinical and procedural characteristics are shown in tables 1 and 2, respectively.

Between October 2011 and April 2013, 277 patients underwent lobectomy, bilobectomy or segmentectomy for cancer in a single institution, of whom $65 \%$ were treated by VATS and $35 \%$ by open thoracotomy. A persistent air leak eligible for the study occurred in $12(4.3 \%)$ patients. Two patients were eligible but were not included as they declined study participation. 10 patients with evidence of an air leak $>100 \mathrm{~mL} \cdot \mathrm{min}^{-1}$ at mean \pm SD post-operative day $10 \pm 3$ were included and evaluated for air leak closure during a diagnostic balloon occlusion test bronchoscopy. Demographic characteristics of all patients who entered the study are presented in table 1. Procedure related characteristics on the day of IBV treatment are listed in table 2. The median duration of the post-operative air leak before valve treatment was 7 days (range 7-13 days), the median air leakage was $490 \mathrm{~mL} \cdot \mathrm{min}^{-1}$ and a median number of four IBV valves was implanted. One patient (thoracotomy right upper lobe plus right middle lobe) was not scheduled for IBV treatment under general anaesthesia. This patient had a calculated predicted post-operative (ppo) forced expiratory volume in $1 \mathrm{~s}$ (FEV1) of $37 \%$ and ppo diffusing capacity of the lung for carbon monoxide of $46 \%$ for right sided

\section{TABLE 1 Demographic data of patients that entered the study}

\section{Outcome}

Males/females
Age years
Anatomical resection lung cancer/metastasis
COPD
FEV $1 \%$ pred
ppoFEV $1 \%$ pred
DLCO \% pred
ppoDLCO \% pred

$9 / 1$

$67(46-75)$

$9 / 1$

7

79 (41-97)

$66(35-87)$

$71(58-80)$

$60(43-67)$

Data are presented as $\mathrm{n}$ or median (range). COPD: chronic obstructive pulmonary disease; FEV1: forced expiratory volume in $1 \mathrm{~s}$; ppo: predicted post-operative; DLCO: diffusing capacity of the lung for carbon monoxide. " : Tiffeneau index $<0.70$. 
bilobectomy superior, and experienced suffocation during the diagnostic balloon occlusion test of the remaining lower lobe bronchus, while a selective more distal occlusion did not result in air leak cessation. In addition, this patient's pre-operative perfusion scan demonstrated a $67 \%$ perfusion to the right lung. Thus, in intention to treat, $90 \%$ of the patients were scheduled for intrabronchial valve treatment.

\section{Outcome measures}

Outcome measures are presented in tables 3-5.

The primary study outcome measurement demonstrated a median air leak cessation at 2 days after valve treatment, which resulted in chest tube removal in the patients receiving valves at a median of 4 days (range 1-14 days) after valve placement (table 3).

On day 1 after valve placement, the air leak was reduced by $90-100 \%$ in six patients, while in three patients a reduced air leak recurrence (defined as $<50 \%$ of its initial value) was observed despite the fact that nearly complete air leak cessation had been demonstrated at valve implantation under general anaesthesia (table 4). In these patients, a minimal IBV displacement (without migration) was documented during flexible bronchoscopy under local anaesthesia. Shallow depth of the target bronchus was judged to be the main reason for these displacements: the anchor points were positioned in a more distal bronchus, resulting in suboptimal axis of the valves and inadequate fitting of the valve umbrella in the targeted bronchus. These patients were discharged (two on day 3 and one on day 7 after valve treatment) with a Heimlich valve connected to their chest tube. In these three patients, the chest drain could be removed at day 14 after intrabronchial valve treatment and an additional surgical intervention was not required.

During the entire study, no deaths, no cardiovascular complications and no implant-related events, such as infection distal to the IBV, lobar atelectasis, haemoptysis, persistent cough, pneumothorax or expectoration of a valve, occurred. One patient (patient 10) suffered from respiratory insufficiency requiring noninvasive positive-pressure ventilation during 2 weeks until valve removal. This patient had a calculated ppoFEV1 of $35 \%$ and developed a massive prolonged air leak with subcutaneous emphysema after an upper lobe lobectomy, requiring intrabronchial valve treatment of almost the entire lower lobe.

All patients underwent spirometry a few hours before valve removal and a follow-up spirometry within a week of valve removal allowing calculation of the magnitude of lung function alteration associated with valve occlusion (table 5). A significant decrease in FEV1 was found at airway closure by valve implantation (mean FEV1 53\% versus $61 \%$ predicted; $\mathrm{p}=0.0002$ ). A $5-10 \%$ decrease in $\mathrm{FEV}_{1} \%$ predicted was observed in patients when a right upper lobe was treated with intrabronchial valves, while a $10-15 \%$ decrease in FEV $1 \%$ predicted was observed when a lower lobe was treated with intrabronchial valves. The removal of the IBVs was performed at a median of 23 days (range 14-28 days) (table 3). In one patient (patient 10), valve removal was performed earlier, at day 14 , as valve treatment induced respiratory insufficiency. No patient

\begin{tabular}{|c|c|c|c|c|c|c|c|}
\hline 1 & VATS lobectomy RLL & 7 & 2000 & 0 & Yes & RB 1-2-3 & 4 \\
\hline 2 & VATS lobectomy RLL & 7 & 1200 & 0 & Yes & RB 1-2-3 & 4 \\
\hline 5 & $\begin{array}{c}\text { VATS segmentectomy } \\
\text { apex RUL }\end{array}$ & 13 & 360 & 31 & No & RB 2-3 & 2 \\
\hline 6 & VATS lobectomy RUL & 13 & 180 & 55 & Yes & RB 6 & 4 \\
\hline 7 & VATS lobectomy RUL & 7 & 180 & 60 & Yes & RB 6-7-8-9-10 & 5 \\
\hline 8 & Thoracotomy LUL & 7 & 720 & 0 & Yes & LB 8-9-10 & 9 \\
\hline 9 & Thoracotomy LUL & 8 & 2500 & 20 & Yes & LB 6-9-10 & 6 \\
\hline
\end{tabular}

SubQ Em: subcutaneous emphysema (assessed on chest radiograph); VATS: video-assisted thoracic surgery; RLL: right lower lobe; RUL: right upper lobe; RML: right middle lobe; LUL: left upper lobe; RB: right segmental bronchus; LB: left segmental bronchus; NA: not applicable. \#: measured on chest radiograph as distance between cupola and apex of the lung; ": using intrabronchial valve (IBV Valve System; Olympus Respiratory America, Redmond, WA, USA). 


\section{TABLE 3 Characteristics during follow-up}

$\begin{array}{lll}\text { Patient } & \text { Air leak days } & \text { Chest tube days }\end{array}$

\begin{tabular}{lccc}
\hline $\mathbf{1}$ & 2.0 & 4 & 21 \\
$\mathbf{2}$ & 3.5 & 5 & 28 \\
$\mathbf{3}$ & 0.5 & 3 & 21 \\
$\mathbf{5}$ & 0.0 & 1 & 24 \\
$\mathbf{6}$ & $3.0^{\#}$ & 14 & 27 \\
$\mathbf{7}$ & $3.0^{\#}$ & 14 & 23 \\
$\mathbf{8}$ & $7.0^{\#}$ & 14 & 28 \\
$\mathbf{9}$ & 0.0 & 4 & 18 \\
$\mathbf{1 0}$ & 0.0 & 4 & 14 \\
Median & 2.0 & 4 & 23 \\
\hline
\end{tabular}

IBV: intrabronchial valve. \#: documented dislocation of one endobronchial valve was responsible for recurrence of a reduced air leak; these patients were discharged with a Heimlich valve.

developed a pneumothorax after elective valve removal. Recurrence of air leak associated with valve displacement in three patients was not associated with delayed valve removal beyond the pre-planned period.

The cost of one IntraBronchial Valve is $€ 1500$, while the cost of instruments for placement is $€ 970$ ( $€ 600$ for the deployment catheter and loader, $€ 200$ for the IBV airway sizing kit and $€ 170$ for the balloon catheter). The median direct cost related to valve management was $€ 6970$ (range $€ 2470-14470$ ) per patient.

\section{Discussion}

This is the first prospective study to evaluate the efficacy of IBV treatment for a prolonged pulmonary air leak in a well-defined patient group after anatomical lung resections for cancer using accurate measurements of the expiratory pulmonary air leak. The treatment approach and algorithm based on quantitative air leak monitoring lead to successful air leak cessation at a median of 2 days after valve therapy and chest tube removal at a median of 4 days after valve therapy. Overall, a Heimlich valve could be avoided in six out of 10 patients. Moreover, we could prove a safe valve removal 3 weeks after valve therapy. This interval is feasible, as a visceral pleural tear will be epithelialised after 3 weeks.

A post-operative pulmonary air leak after an anatomical pulmonary resection is usually managed conservatively, such as a longer period of chest tube drainage or the use of a Heimlich valve. However, it must be stressed that other different strategies have also been used and no unique algorithm has been validated [9]. There is no standard definition for a persistent pulmonary air leak in the literature, but an air leak has been considered to be a complication only when it persists beyond the normal hospital stay. The median hospital stay after a lobectomy is 4-7 days and, therefore, a prolonged persistent pulmonary air leak could be defined as one that is still present on post-operative day 7. Data from the European Society of Thoracic Surgeons 2012 database show that the percentage of air leaks present on day 5 is $8.3 \%$ for lobectomy, $6.8 \%$ for segmentectomy and $11.1 \%$ for bilobectomy [10]. Apart from a prolonged hospital

$$
\text { TABLE } 4 \text { Quantitative evolution of the air leak from day (d) }-1 \text { until day }+4^{\#}
$$

\begin{tabular}{|c|c|c|c|c|}
\hline Patient & $\begin{array}{l}\text { Air leak d-1 } \\
\mathrm{mL} \cdot \mathrm{min}^{-1}\end{array}$ & $\begin{array}{l}\text { Air leak } \mathrm{d}+0 \\
\mathrm{~mL} \cdot \mathrm{min}^{-1}\end{array}$ & $\begin{array}{l}\text { Air leak d+1 } \\
\mathrm{mL} \cdot \mathrm{min}^{-1}\end{array}$ & $\begin{array}{c}\text { Air leak d+4 } \\
\mathrm{mL} \cdot \mathrm{min}^{-1}\end{array}$ \\
\hline 1 & 2000 & 100 & 130 & 0 \\
\hline 2 & 1200 & 20 & 100 & 0 \\
\hline 3 & 500 & 20 & 0 & 0 \\
\hline 5 & 360 & 0 & 0 & 0 \\
\hline 6 & 180 & 30 & 90 & $30^{\circ}$ \\
\hline 7 & 180 & 10 & 80 & $50^{\circ}$ \\
\hline 8 & 720 & 30 & 200 & 50 \\
\hline 9 & 2500 & 0 & 0 & 0 \\
\hline 10 & 480 & 0 & 0 & 0 \\
\hline
\end{tabular}

\footnotetext{
${ }^{\#}: \mathrm{d}+0=$ day of intrabronchial valve placement. ${ }^{\top}$ : patients 6 and 7 were discharged with a Heimlich valve on day +3 .
} 
TABLE 5 Functional consequences of intrabronchial valve removal

\begin{tabular}{lcccc} 
Patient & Target lobe & FEV $\mathbf{\%}$ pred $^{\#}$ & FEV $\mathbf{\%}$ pred $^{\boldsymbol{\pi}}$ & $\Delta$ FEV $\mathbf{\%}$ \\
\hline $\mathbf{1}$ & RUL & 56 & 67 & 11 \\
$\mathbf{2}$ & RUL & 71 & 78 & 7 \\
$\mathbf{3}$ & Apex RLL & 60 & 61 & 1 \\
$\mathbf{5}$ & RUL & 48 & 53 & 5 \\
$\mathbf{6}$ & Apex RLL & 67 & 75 & 8 \\
$\mathbf{7}$ & RLL & 47 & 60 & 13 \\
$\mathbf{8}$ & LLL & 56 & 70 & 14 \\
$\mathbf{9}$ & LLL & 49 & 58 & 9 \\
$\mathbf{1 0}$ & RLL & 19 & 28 & 9
\end{tabular}

FEV1: forced expiratory volume in $1 \mathrm{~s} ; \Delta$ : change; RUL: right upper lobe; RLL: right lower lobe; LLL: left lower lobe. \#: spirometry performed before valve removal (on the day of valve removal, before the procedure); ': spirometry performed within a week after valve removal.

length of stay and increased morbidity or post-operative complications, a persistent post-operative pulmonary air leak may also impede the opportunity of adjuvant chemotherapy in cases where this is indicated.

Our study conclusions support the earlier retrospective series suggesting that bronchial valve therapy using the IBV Valve System for prolonged air leak has an acceptable safety and efficacy for the treatment of a prolonged pulmonary air leak [6,7]. Accordingly, in 2008, the US Food and Drug Administration (FDA) approved the IBV Valve System for use in the treatment of prolonged air leaks of the lung, or significant air leaks that are likely to become prolonged air leaks following lobectomy, segmentectomy or lung volume reduction surgery [11]. There are, however, several clinical differences that can be appreciated compared with prior series (table 6). We decided to restrict the indication for valve treatment to patients with a persistent air leak after an anatomical pulmonary resection (as suggested by FDA approval) and, thus, did not include patients who had an air leak caused by a spontaneous pneumothorax or a pleural decortication procedure (e.g. for empyema or mesothelioma). This choice might explain the fact that a higher candidature rate for valve treatment was observed (90\% versus $80 \%)$ and a higher rate of successful chest tube removal was observed (100\% versus 63-75\%). Our patients might have had a "more localised" pleural injury and/or better pulmonary function and, thus, respiratory reserve, as they were previously considered medically fit to undergo a pulmonary resection. We were also able to further reduce the time until chest tube removal from a median of 1-2 weeks to a median of 4 days. This can be explained by the use of a digital thoracic drainage system, which enables continuous monitoring of the air leak not only helpful for appropriate valve placement but also for more precise timing of chest drain removal [12-14]. Finally, previous reports reevaluated patients $\sim 6$ weeks after valve placement to determine whether the valve removal is a feasible option. We clearly demonstrated an uneventful valve removal 3 weeks after valve placement in all patients.

TABLE 6 Comparison of published studies on bronchial valve treatment for persistent pulmonary air leak

GilLesPie et al. [6]

FIRLINGER et al. [7]

Current study

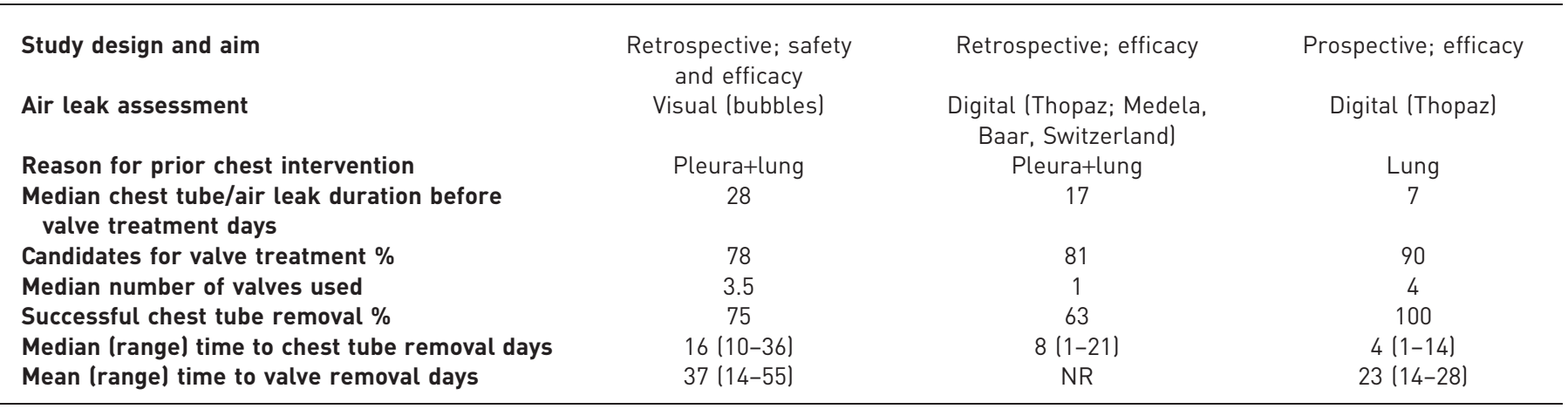

NR: not reported. 
Decreased FEV1 suggests that respiratory compromise associated with airway closure may be a potential side-effect of air-leak treatment with IBVs, in particular when this leak complicates lobectomy. This drawback seems limited, as the initial diagnostic balloon occlusion test may allow for exclusion of the more disabled patients and, thereby, limit the number of respiratory failures. In addition, valve treatment is an easily reversible procedure offering a quick answer in case of significant post-treatment respiratory insufficiency; this was the case in patient 10, from whom we decided to remove the valves after 14 days as this was considered to be enough time for pleural re-epithelialisation. Finally, no atelectasis was observed after valve implantation and no other valve-related complication was observed. Altogether, these observations allow us to conclude that this procedure is safe.

This study has the following potential limitations. This was a prospective nonrandomised study. Thus, the true benefit compared with any other standard approach (e.g. watchful waiting, ambulatory Heimlich valve or surgical intervention) or a cost-effectiveness analysis has not yet been assessed. In addition, a true quantitative criterion (duration and quantity) for a prolonged air leak requiring further intervention does not exist. Our inclusion criteria seem acceptable, as patients with failure of valve treatment (i.e. finally requiring a Heimlich valve) were in fact those with the smallest prolonged air leak. Furthermore, while the valve treatment was performed under general anaesthesia with positive pressure ventilation, we observed a slight valve displacement in three patients the day after the procedure. This valve displacement caused a recurrence of the air leak to a lesser extent than was the case before valve placement ( $\geqslant 50 \%$ reduction), resulting in a short period of ambulatory Heimlich valve treatment. Finally, the number of patients seems limited, but it must be stressed that a prolonged air leak fortunately remains an uncommon complication ( $4.3 \%$ in our cohort of anatomical pulmonary resections for cancer) and we believe that a larger number of patients would not significantly change our results.

In conclusion, the application of a digital thoracic drainage assessment of the pulmonary air leak might guide intrabronchial valve placement and allow a safe fast-tracking chest tube removal in patients with a persistent air leak after a pulmonary resection for cancer. Air leak closure can be obtained with minimal airflow alteration by intrabronchial valves leading to chest tube removal in all patients.

\section{References}

Farjah F, Wood D, Yanez D 3rd, et al. Temporal trends in the management of potentially resectable lung cancer. Ann Thorac Surg 2008; 85: 1850-1855.

2 Varela G, Jimenez M, Novoa N, et al. Estimating hospital costs attributable to prolonged air leak in pulmonary lobectomy. Eur J Cardiothorac Surg 2005; 27: 329-333.

3 Brunelli A, Xiume F, Al Refai M, et al. Air leaks after lobectomy increase the risk of empyema but not of cardiopulmonary complications: a case-matched analysis. Chest 2006; 130: 1150-1156.

4 Toma T, Kon O, Oldfield W, et al. Reduction of persistent air leak with endoscopic valve implants. Thorax 2007; 62: 830-833.

5 Travaline J, McKenna R, de Giacomo T, et al. Treatment of persistent air leaks using endobronchial valves. Chest 2009; 136: 355-360.

6 Gillespie CT, Sterman DH, Cerfolio RJ, et al. Endobronchial valve treatment for prolonged air leaks of the lung: a case series. Ann Thorac Surg 2011; 91: 270-273.

7 Firlinger I, Stubenberger E, Müller M, et al. Endoscopic one-way valve implantation in patients with prolonged air leak and the use of digital air leak monitoring. Ann Thorac Surg 2013; 95: 1243-1250.

8 Dooms C, De Leyn P, Yserbyt J, et al. Endobronchial valves for persistent postoperative pulmonary air leak: accurate monitoring and functional implications. Respiration 2012; 84: 329-333.

9 Venuta F, Rendina E, De Giacomo T, et al. Postoperative strategies to treat permanent air leaks. Thorac Surg Clin 2010; 20: 391-397.

10 European Society of Thoracic Surgeons Database. Annual report 2012. www.ests.org/_userfiles/pages/files/ Database\%20ESTS_Report_2012.pdf Date last updated: May 2012. Date last accessed: November 18, 2013.

11 US Food and Drug Administration. News and events. FDA approves lung valve to control some air leaks after surgery. Date issued: October 24, 2008. www.fda.gov/NewsEvents/Newsroom/PressAnnouncements/2008/ucm116970. htm Date last updated: April 12, 2013. Date last accessed: November 18, 2013.

12 Cerfolio R, Bryant A. The benefits of continuous and digital air leak assessment after elective pulmonary resection: a prospective study. Ann Thorac Surg 2008; 86: 396-401.

13 Brunelli A, Salati M, Refai M, et al. Evaluation of a new chest tube removal protocol using digital air leak monitoring after lobectomy: a prospective randomised trial. Eur J Cardiothorac Surg 2010; 37: 56-60.

14 Filosso P, Ruffini E, Solidoro P, et al. Digital air leak monitoring after lobectomy for primary lung cancer in patients with moderate COPD: can a fast-tracking algorithm reduce postoperative costs and complications? J Cardiovasc Surg 2010; 51: 429-433. 